\title{
Image-based correlation between the meso-scale structure and deformation of closed-cell foam
}

\author{
Yongle Sun ${ }^{1 *}$, Xun Zhang ${ }^{2}$, Zhushan Shao ${ }^{3}$, Q.M. Li $^{1,4}$ \\ ${ }^{1}$ School of Mechanical, Aerospace and Civil Engineering, The University of Manchester, \\ Sackville Street, Manchester M13 9PL, UK \\ ${ }^{2}$ Henry Moseley X-ray Imaging Facility, School of Materials, The University of Manchester, \\ Upper Brook Street, Manchester M13 9PL, UK \\ ${ }^{3}$ School of Civil Engineering, Xi'an University of Architecture \& Technology, Xi'an \\ 710055, China \\ ${ }^{4}$ State Key Laboratory of Explosion Science and Technology, Beijing Institute of Technology, \\ Beijing 100081, China
}

\begin{abstract}
In the correlation between structural parameters and compressive behaviour of cellular materials, previous studies have mostly focused on averaged structural parameters and bulk material properties for different samples. This study focuses on the meso-scale correlation between structure and deformation in a 2D foam sample generated from a computed tomography slice of Alporas ${ }^{\mathrm{TM}}$ foam, for which quasi-static compression was simulated using 2D image-based finite element modelling. First, a comprehensive meso-scale structural characterisation of the 2D foam was carried out to determine the size, aspect ratio, orientation and anisotropy of individual cells, as well as the length, straightness, inclination and thickness of individual cell walls. Measurements were then conducted to obtain the axial distributions of local structural parameters averaged laterally to compression axis. Second, the meso-scale deformation was characterised by cell-wall strain, cell area ratio, digital image correlation strain and local compressive engineering strain. According to the results, the through-width sub-regions over an axial length between the average (lower bound) and the maximum (upper bound) of cell size should be used to characterise the meso-scale heterogeneity of the cell structure and deformation. It was found that the first crush band forms in a sub-region where the ratio of cell-wall thickness to cell-wall length is a minimum, in which the collapse deformation is dominated by the plastic bending and buckling of cell walls. Other morphological parameters have secondary effect on the initiation of crush band in the 2D foam. The finding of this study suggests that the measurement of local structural properties is crucial for the identification of the "weakest" region which determines the initiation of collapse and hence the corresponding collapse load of a heterogeneous cellular material.
\end{abstract}

\footnotetext{
* Corresponding author.

E-mail address: yongle.sun@manchester.ac.uk; sunyongletl@gmail.com (Y.L. Sun)
} 
Keywords: Aluminium foam; cell structure; crushing; computed tomography; image-based modelling

\section{Introduction}

Cellular materials, such as foams and honeycombs, are being increasingly used in transport, aerospace, civil and biomedical engineering fields due to their unique mechanical, thermal and electromagnetic properties $[1,2]$. Their compressive properties are particularly important for their applications as energy absorbers and load attenuators [3]. At least three spatial scales are manifest in cellular materials. At the macro-scale, a cellular material is usually treated as a "homogeneous" material and the continuum approach is adopted. At the meso-scale, the cell structure constructing the cellular material plays key roles in determining the macroscopic material properties. At the micro-scale, the chemical composition and microstructure of the constituent material itself are important. This study is aimed at correlating the meso-scale structure with the deformation of a closed-cell foam subjected to uniaxial compression.

For a closed-cell foam, the constituent cells are separated from each other by walls, facilitating the identification of individual cells for geometrical measurement. The size, orientation and anisotropy of the cells are parameters of major interest, which, however, are not easy to measure when cells are irregular. An equivalent dimension is often used to represent the cell size. For instance, Saadatfar et al. [4] used the equivalent diameter of a sphere whose volume is equal to the cell volume to indicate the cell size. The characterisations of the orientation and anisotropy of cells are usually based on equivalent or best-fit ellipsoids (ellipses in a 2D case). Consequently, the orientation can be simply defined as the angle between the major axis of the equivalent ellipsoid (or ellipse) and a reference axis [5, 6]. The quantification of cell anisotropy is more complicated. Benouali et al. [5] proposed that the eigenvalues of the $3 \mathrm{D}$ inertia matrix of the equivalent ellipsoids can be used to evaluate the cell anisotropy. Simone and Gibson [6] used the ratios of the principal values of a fabric tensor which is formed using best-fit ellipses in three orthogonal planes to estimate the anisotropies in different planes.

For the constituent walls of a cell, the curvature, corrugation and thickness are important parameters. The definition of curvature can be based on the cell wall and its chord connecting its end nodes [7]. The corrugation is characterised by its amplitude and number in a wall [7]. The cell-wall thickness is not straightforward to quantify if the boundaries of the wall are not parallel. Němeček et al. [8] simply used the minimum distance between two neighbouring cells to estimate the cell-wall thickness, which, however, cannot capture the thickness variation 
along the wall. The method proposed by Hildebrand and Rüegsegger [9], which defines local thickness as the diameter of the largest circle containing the material point and being completely inside the wall, can be applied to capture the thickness variation, but few results based on this method have been reported for foams.

Meso-scale inhomogeneous deformations, such as shear banding [10, 11], localised crushing $[12,13]$ and progressive folding $[14,15]$, are common in cellular materials under compression. There are several methods to quantify the localised deformation at the meso-scale. Digital image correlation (DIC) has been increasingly used to calculate local strain fields [16-18]. In DIC the acquired images are divided into subsets (i.e. interrogation windows) before correlation. Then the displacement is calculated by maximising the correlation coefficient for each interrogation window between successive images, from which the strain field can be computed [19-21]. In numerical simulations, the nodal displacements can be used to calculate local deformation directly. For instance, Zou et al. [22] defined the local engineering strain using the relative displacement between two neighbouring cross-sections with different averaged nodal displacements. Liao et al. [23] adopted a local strain calculation method based on selected discrete cell-wall nodes within a circular neighbourhood in a finite element model. Among these methods, DIC has most general applicability, but it has rarely been used to analyse the results of simulations.

The effects of the structural parameters of cells and their walls on macroscopic properties (e.g. compressive strength) have been extensively studied. However, in most cases, the structural parameters, such as the relative density [1,3], the size and shape irregularity of cells [5, 24-29], and the dimension and imperfection of cell walls [24, 30, 31], were averaged with regard to the whole sample for evaluation, which cannot be used to establish the relationship between macro-scale properties and meso-scale structural parameters (e.g. local density, local cell size and local cell-wall thickness) of a heterogeneous foam material. To define "weak" cells susceptible to premature collapse, Bart-Smith et al. [18] proposed two criteria based on cell morphology, i.e. (1) ellipsoidal cells with T-shaped wall intersections and (2) cells with appreciably curved walls. As for the effect of cell size on deformation, McDonald et al. [32] observed that strain concentration in isolated large cells (i.e. those with small surrounding cells) is more significant than in an aggregate of large or moderately sized cells. However, the effect of cell size on compressive strength seems controversial, as some experimental observations $[25,33]$ show that the larger the cell size, the larger the compressive strength, while other observations [26, 34] indicate the opposite. Regarding other morphological parameters, it has been shown that the orientation and anisotropy of cells can also affect the compressive 
deformation and strength of closed-cell foams $[4,5]$. The effect of cell-wall thickness, despite its importance for bending-dominated foams, has been rarely investigated.

The previous studies have shed some light on the effect of the aforementioned structural parameters on the compressive behaviour of cellular materials. However, the axially distributed local structural parameters, in contrast to the structural parameters for a bulk foam sample or the individual cells (or cell walls), have been rarely examined to disclose their roles in the determination of the macro-scale collapse stress and meso-scale crushing deformation of a heterogeneous cellular material.

In this study, a 2D finite element (FE) model of a virtual foam has been generated from a computed tomography (CT) slice of an aluminium closed-cell foam sample (Alporas ${ }^{\mathrm{TM}}$, which was regarded with least heterogeneity [35] among many types of foams). The meso-scale structure of this $2 \mathrm{D}$ foam was then characterised in detail including the size, aspect ratio, orientation and anisotropy of cells, and the length, straightness, inclination and thickness of cell walls. The local structural parameters and their axial distributions were obtained in through-width sub-regions perpendicular to the compression axis in a virtual quasi-static compression test performed by image-based FE modelling. Furthermore, the meso-scale deformation of the 2D foam was characterised by cell-wall strain, cell area ratio, digital image correlation (DIC) strain, and local compressive engineering strain (defined using relative cross-sectional displacement). Finally, the correlation between meso-scale structure and deformation was established and discussed.

\section{Material and method}

\subsection{Two-dimensional foam}

A $2 \mathrm{D}$ foam $(30 \times 30 \mathrm{~mm})$ was generated from the diametric cross-section of a cylindrical sample of a closed-cell aluminium foam (Alporas ${ }^{\mathrm{TM}}$ ) using computed micro-tomography, and the details of the X-ray imaging can be found in Ref. [36]. The sample size is sufficient to obtain representative bulk material properties [37].

In comparison with idealised cell structures such as regular hexagonal-cell and circular-cell honeycombs [38] and random Voronoi foams [27], the 2D foam model based on the cross-sectional geometry of an actual foam has advantages owing to its capability of incorporating a realistic cell structure. This is important for establishing structure-property relationships for real cellular materials. Fig. 1 shows the cell structure of the $2 \mathrm{D}$ foam. It is 
evident that the cell morphology and topology are complicated, and the prevalent idealised foam models such as Kelvin and Voronoi models [39, 40] cannot capture well these structural features. In addition, cell-wall imperfections, e.g. curvature, corrugation, damage (or partial missing), and nonuniform thickness, are clearly seen in Fig. 1, which are related to the liquid-state foaming process of the Alporas ${ }^{\mathrm{TM}}$ foam [6].

The 2D foam has a relative density (i.e. area ratio between the cell walls and the bulk foam sample) of $12.5 \%$. Fig. 2a shows the constituent cells (148 cells in total of which 111 are fully inside the sample). In order to evaluate the meso-scale heterogeneity of the cell structure along the compression axis (vertical direction), structural parameters are averaged across the sample width (laterally) and over a vertical sampling length, $l,(0<l<30 \mathrm{~mm}$, see Fig. 2a). The position of the lateral through-width sub-region is indicated by the $\mathrm{Y}$ coordinate of the horizontal central line of the sub-region. Adjacent sub-regions overlap and the incremental distance for the vertical position is equal to one pixel size (i.e. $0.019 \mathrm{~mm}$ ). The bulk measure is obtained when the sampling length is equal to the sample size $(l=30 \mathrm{~mm})$.

Fig. $2 b$ shows the skeletonised foam which is used to extract structural parameters describing the cell walls. The skeletonisation was performed using open-source code ImageJ, and basically, the algorithm $[41,42]$ thins each wall into its medial axis and then constructs a skeleton of single pixel thickness. This preserves the topological properties of the original cell structure and facilitates the characterisation of each wall. The skeletonised cell structure has 411 branches (i.e. skeletonised walls), based on which, the cell-wall length $s$, the node-to-node distance (or chord length) $w$, and the inclination angle $\alpha$ between the chord and the vertical compression axis, can be measured, as shown in Fig. 2 b. The ratio $w / s$ of a cell-wall can be used as a metric quantifying the cell-wall straightness. A straight wall has $w / s=1$, while the smaller the ratio, the more the wall deviates from a straight line.

Fig. 3a illustrates the local cell-wall thickness at a material point (a pixel in a digital image), which is defined as the diameter of the largest circle which contains the material point and is completely inside the wall [9]. The aspect ratio of a cell is defined using the circumscribed rectangle, as shown in Fig. 3b. The measurements of orientation and anisotropy are based on an ellipse which has the same second-moment as the cell, as shown in Fig. 3c. The orientation is defined as the angle between the major axis of the ellipse and the vertical axis. The anisotropy is simply defined as the ratio of the length difference between the major axis and the minor axis to the length of the major axis, i.e. (a-b)/a. These geometrical parameters were determined using the function of "regionprops" in Matlab (Mathworks, 2014). 


\subsection{Simulated compression test}

A finite element model (FEM) taking account of large deformation effect was developed using commercial code Abaqus/Explicit to simulate the quasi-static compression $\left(\dot{\varepsilon}=1 \times 10^{-3} \mathrm{~s}^{-1}\right)$ of the 2D foam. The Abaqus/Explicit was chosen due to its capability of handling problems with severe nonlinearity (e.g. plasticity) and discontinuity (e.g. contact). ScanIP (Simpleware Ltd, UK) was used to directly convert the CT image into a high-quality FE mesh [43]. A plane strain assumption was adopted and 398703 linear elements were used, as shown in Fig. 4. The aluminium cell-wall material was taken to have elastic modulus, Poisson's ratio, yield strength and density of $68 \mathrm{GPa}, 0.33,172 \mathrm{MPa}$ and $2710 \mathrm{~kg} / \mathrm{m}^{3}$, respectively, and an elastic, perfectly plastic material model was used.

Two rigid platens (not shown in Fig. 4) were used to impose the compressive loading on the two ends of the 2D foam sample. A downward displacement was applied to the top platen, while a constraint of all degrees of freedom was applied to the bottom platen. Frictionless contact was defined for all potentially touching parts of the model, and the foam sample was free from any lateral constraint. The nominal stress $\sigma$ was obtained as the reaction force on the platen divided by the original sample width. The foam underwent a macroscopically uniaxial compression process. Intuitively, the cross-section motion can be used to characterise the deformation under 1D loading. Therefore, a "particle" array approach was adopted [22, 36], which defines a line of "particles" along the compression direction to represent the average motion of 79 equally spaced cross-sections through the foam, as illustrated in Fig. 4.

The nominal strain $\varepsilon$ was obtained as the vertical displacement of the top platen divided by the original sample height, while the meso-scale deformation of the $2 \mathrm{D}$ foam under compression was characterised by different methods. For cell deformation, the area strain was obtained as the ratio of the area of the deformed cell to its original value, the local engineering strain was taken to be the shortening of two cross-sections divided by their original distance, the calculation of digital image correlation (DIC) strain was based on the displacement gradient obtained using image subsets having different window sizes. For cell-wall deformation, the true strain at the integration point in each finite element was used to represent the deformation at the material point in the cell walls. 


\section{Results}

\subsection{Meso-scale structural characteristics}

Fig. 5 shows the histogram of cell size which is defined as the diameter of a circle having same area as the cell. It is interesting to see that two peak frequencies appear in the histogram, corresponding to the cell sizes of $0.3 \mathrm{~mm}$ and $2.1 \mathrm{~mm}$, respectively, indicating a bimodal distribution of cell size. Similar bimodal cell size distribution was also observed in the 3D counterpart of the 2D foam, i.e. Alporas ${ }^{\mathrm{TM}}$ foam [4], as shown in Fig. 5. This similarity implies that the 2D image-based foam can well represent an actual foam material in this aspect. The difference in the absolute frequency values between the $2 \mathrm{D}$ foam and the $3 \mathrm{D}$ foam can be attributed to three factors, i.e. (i) the cell structures were taken from different Alporas ${ }^{\mathrm{TM}}$ foam samples, (ii) the cell size based on a cross-section of a 3D sample cannot completely represent the cell size based on the 3D sample, and (iii) different bin widths may be adopted in the plots of the histograms. Nevertheless, in general, the bimodal distribution of cell size inherent in a 3D sample should be reflected by the dimension characteristics of cross-sectional cells of the 3D sample, as long as the cells in the foam have no specific orientations and geometric arrangements.

The mean cell size, $d$, is obtained using the following equation

$$
d=\frac{1}{M} \sum_{i=1}^{M} d_{i}
$$

where $d_{i}$ is the size of cell $i$ and $M$ is the total number of cells. For the 2D foam, we have $d=2.05 \mathrm{~mm}$. A similar averaging method is applied to the aspect ratio, orientation and anisotropy of cells, and the length, inclination and straightness of cell walls.

Fig. 6 shows the contours and histograms of the aspect ratio, orientation and anisotropy of all cells. Overall, most of the cells have aspect ratio close to one and do not show any significant preferred orientation. As regards cell anisotropy, the great majority of cells have an anisotropy index smaller than 0.65 , indicating that the cells have a moderate level of anisotropy.

Fig. 7 shows the histograms of $s, w / s$ and $\alpha$. It is seen that $55 \%$ of the cell walls are longer than $1 \mathrm{~mm}$. In general, the walls are close to straight lines, of which $80 \%$ have $w / s>0.9$ (note that a value of 0.64 and 1.00 represents a semi-circle and a straight line, respectively). The 
inclination angle $\alpha$ is relatively uniformly distributed, indicating that the cell walls are not preferentially aligned. This is consistent with the characteristic of cell orientation, see Fig. $6 \mathrm{~d}$.

Fig. 8 shows the contour and histogram of the local cell-wall thickness. In general, the walls are thinnest at the middle-span and thickest at the connecting nodes. This arises from the foaming process for Alporas ${ }^{\mathrm{TM}}$ foam [6]. The average of the local cell-wall thickness [9] is obtained by

$$
h=\frac{1}{N} \sum_{j=1}^{N} h_{j}
$$

where $h_{j}$ is the local cell-wall thickness at pixel $j$ (material point in a continuum sense) and $N$ is the total number of pixels within the region of interest.

Fig. 9 shows the distributions of local relative density and cell-wall thickness averaged laterally to compression axis when different axial sampling lengths are considered. Unsurprisingly, it is clear from Fig. 9 that both relative density and cell-wall thickness are very scattered when $l$ is small (see for example when $l=0.0093 d$ ). The parameters averaged over a sampling length which is much smaller than the mean cell size are physically meaningless for a characterisation of the meso-scale structure in a continuum sense. When the sampling length is equal to the mean cell size $(l=d)$, it is seen that the data scatter is less but still appreciable. The nonuniform axial distributions of the structural parameters indicate the heterogeneous nature of the cell structure. The significant variability of the mechanical properties of Alporas ${ }^{\mathrm{TM}}$ foam [44] also indicates the structural heterogeneity. When the sampling length is further increased to the maximum cell size (i.e. $l=3.65 d$ ) the high-frequency scatter is largely reduced, and it shows that the local relative density and mean cell-wall thickness near the top end of the foam sample are substantially lower than the bulk value. In addition, it is interesting to see that the distribution of the relative density is overall similar to that of the cell-wall thickness, which may not be necessary, as relative density also depends on cell size.

A summary of the meso-scale structural parameters is given in Table 1. The cell size has a high scatter, and its average and maximum can be used as two spatial thresholds for meso-scale structural characterisation. In general, the cells have no evidently preferred orientation and the mean orientation angle is slightly smaller than $45^{\circ}$. The mean aspect ratio is close to one and 
the anisotropy is not very significant. The cell-wall thickness also has a high scatter, and the thickest portion is almost 27 times thicker than the thinnest portion. Moreover, the mean cell-wall thickness is about one fourth of the mean cell-wall length. The cell-wall straightness index has a low scatter and most cell walls are close to straight lines. The cell walls have no preferential direction to align, and the mean inclination angle is close to $45^{\circ}$.

\subsection{Compressive load and deformation}

Fig. 10 shows the nominal stress-strain curve obtained from the quasi-static compression simulation. It shows that the stress increases to a maximum and then remains more or less constant leading to a plateau regime followed by a rapid rise in stress due to global structural densification. This predicted stress-strain relationship qualitatively agrees with the experimental compression results of foam materials $[1,3,7]$. Four nominal strains typical of different stages of compression, i.e. $0.01,0.15,0.30$ and 0.55 , are highlighted to facilitate the correlation between stress level and cell deformation, which will be further discussed below.

Fig. 11 shows the compressive deformation of the $2 \mathrm{D}$ foam at the nominal strains indicated in Fig. 10. The uniaxial and lateral deformations are both heterogeneous even at a relatively small nominal strain of 0.01 , which may not be clear in the $2 \mathrm{D}$ foam configuration but can be clearly seen in the 1D "particle" array shown in Fig. 11a. A crush band, somewhat inclined, initiates near the top end of the foam sample when the strain increases to 0.15, and another band forms approximately in the middle when the strain further increases to 0.30. At these two strains, the foam exhibits more heterogeneous and localised deformation than that at $\varepsilon=0.01$, as shown in Fig. 11a-c, but the nominal stress remains at a similar level (see Fig. 10). For cell walls, local stress concentrates in the materials within the crush bands, as shown in Fig. 11. Plastic hinges are prevalent and randomly distributed. When the nominal strain reaches 0.55 , all cells have been crushed to some extent and further compaction is produced in a relatively uniform and stable way, which corresponds to a stiffening regime in the stress-strain curve and a densification process of the cell structure.

Fig. 12 shows the cell-wall deformation. It is evident that plastic hinges form in critical load bearing cell walls, particularly in the thin mid-span sections. When nominal strain increases, the cell-wall plastic deformation increases and more plastic hinges form. This confirms that the dominant deformation modes of the 2D foam are plastic bending and 
buckling $[22,36]$. In addition, this implies that the moment of inertia of cell walls, which is determined by the cell-wall thickness, and the length of cell walls, which is associated with bending moment, control the load bearing capacity, and thus they play key roles in cell collapse.

To explore the relationship between cell deformation and cell morphology, the shape evolution of typical cells during compression is shown in Fig. 13. Bart-Smith et al. [18] proposed two critical cell morphologies for initiation of cell collapse, i.e. ellipsoidal cells with T-shaped wall intersections and cells with appreciably curved walls. The cells 1-4 (see Fig. 11a for their location and Fig. 6 for their morphological parameters), which are encompassed by the first crush band, indeed satisfy these criteria. However, cells 5 and 6 also satisfy these morphological conditions, but they do not contribute to the formation of the first crush band. This exception is also applicable to some other cells satisfying the critical morphological criteria. Therefore, the criteria based on morphologies of individual cells and their walls may be just one necessary condition for the initiation of a crush band. This is mainly because the formation of a crush band requires that the cells are collectively weak, while such collective susceptibility cannot be described by the morphological parameters of individual cells or their walls. Therefore, it is worth seeking a sufficient condition for the collective cell collapse and its relationship with meso-scale structural parameters.

Fig. 14 shows the contour maps of area ratios of cells at different nominal strains. This confirms the formation of the first crush band and provides a useful quantitative measure of the meso-scale localised deformation of the $2 \mathrm{D}$ foam under uniaxial compression. The maximum area decrease of the cells is 0.33 at a nominal strain of 0.05 , corresponding to a longitudinal strain approximately equal to 0.17 .

Although the nodal displacement of each finite element can be readily obtained for local strain calculation $[22,23]$ in simulations, the displacement of a material point is difficult to track in experiments and the calculation method of meso-scale strain based on nodal displacement is not unique. A general and standard approach for meso-scale strain measurement is thus needed. Nowadays, the DIC method is widely used to quantify the local deformation and it is generally recognised as reliable when properly used. Therefore, a DIC calculation was also performed here using the foam configurations at nominal strains from $0 \%$ 
to $5 \%$ with an increment of nominal strain of $0.5 \%$, which were obtained from the simulation results. Fig. 15 shows the meso-scale deformation represented by the distortion of a regular square grid, and the normal strain field in vertical direction represented as a contour map. The 2D displacement of the grid was calculated using DIC software, DaVis, developed by LaVision, Gottingen, Germany. The DIC algorithm uses fast Fourier transform (FFT) cross-correlation to compare subsets of the successive images [45]. The correlation windows evaluated here are squares of $16 \times 16$ pixel $(0.26 \times 0.26 \mathrm{~mm}), 128 \times 128$ pixel $(2.08 \times 2.08 \mathrm{~mm})$ and $256 \times 256$ pixel $(4.16 \times 4.16 \mathrm{~mm})$. In this test case, the window size close to mean cell size leads to best meso-scale deformation characterisation. When the window size is too small, i.e. $0.26 \times 0.26 \mathrm{~mm}$, cell-wall deformation is tracked rather than the meso-scale deformation on cell level; whereas when window size is too large, i.e. $4.16 \times 4.16 \mathrm{~mm}$, some desired meso-scale details are lost. More importantly, the maximum local strain $(\sim 0.15$ at $\varepsilon=0.05)$ in the first crush band obtained by DIC using a window size of $2.08 \times 2.08 \mathrm{~mm}$ is comparable to the maximum local engineering strains obtained from the area ratios (see Fig. 14) and the cross-sectional displacements (presented below).

Fig. 16 shows the local engineering strain obtained as the relative cross-sectional displacement divided by original cross-sectional distance, in the through-width sub-regions over axial lengths equal to the average and the maximum of cell size. It appears that the cross-sectional strain oscillates significantly when $l=d$, implying a significant heterogeneity of meso-scale compressive deformation. When the axial length is increased to the maximum cell size, i.e. $l=3.65 d$, the oscillation is largely reduced and the high strain level appears near the top end of the foam sample. Strictly speaking, the local engineering strain is essentially approximate since the original planar cross-sections become distorted when crushing occurs, but it is capable of capturing the location of a crush band and the local strain magnitude in the band, and the measure using this approach is consistent with the compressive strain derived from cell area ratio and the DIC strain results.

\section{Discussion}

The comprehensive characterisations of the cell structure and local deformation, as shown in Figs. 5-9 and Figs. 11-16, provide the basis to correlate the meso-scale structure with the deformation of the $2 \mathrm{D}$ foam subjected to macroscopically uniaxial compression. It is also 
important to note that the average and the maximum of cell size can be used as two spatial thresholds based on which the meso-scale heterogeneities of the structure and deformation of a foam material can be characterised. For the meso-scale structure characterisation, when the axial length of the through-width sub-regions falls in the range between the average and the maximum of cell size (see Fig. 9), the axial nonuniform distributions of structural parameters (e.g. relative density and cell-wall thickness) indicate the meso-scale structural heterogeneity. For the meso-scale deformation characterisation, the local strain field can be well captured by DIC when the window size is close to the mean cell size, while the local engineering strain calculated from relative cross-sectional displacement is representative when the sub-region has an axial length equal to the maximum cell size.

From Fig. 11 and Figs. 14-16, it is seen that the first crush band forms near the top end of the foam sample. Previous studies [18, 32] considered the structural characteristics of individual cells and their walls, but they are insufficient to establish the critical condition for the initiation of a crush band, see Fig. 13 and the associated discussion in Section 3.2. By contrast, the axial distributions of local structural parameters (averaged in the through-width sub-regions) are more representative to indicate the regions where the cells or their walls are collectively weak and hence susceptible to collapse during uniaxial compression. Therefore, they are used here to explore the sufficient condition for the initiation of crushing. Fig. 9, Fig. 17 and Fig. 18 show the axial distributions of the measured structural parameters. The cells in the top part have slightly lower orientation angles, while the cell walls have relatively uniformly distributed inclination angles. The distribution of the aspect ratio is also relatively uniform, except at sample ends. These relatively uniformly distributed structural parameters cannot be correlated with the initiation of crushing. Although it is generally agreed that cells have higher strength when their anisotropy is larger [1,46] or the cell walls are straighter [30], Fig. 17d and Fig. 18d show that the location where first crush band initiates actually corresponds to relatively high cell anisotropy and cell-wall straightness. This indicates that these two morphological parameters are also secondary for the initiation of the crush band in the $2 \mathrm{D}$ foam.

Other structural parameters, such as cell size, cell-wall length, cell-wall thickness and relative density, are all significantly nonuniformly distributed. The cell size and the cell-wall 
length exhibit similar distributions, although the former is overall larger than the latter. The cell size and cell-wall length are both largest in the top half of the sample. The local relative density and cell-wall thickness are lowest near the top end of the sample, see Fig. 9. For these structural parameters, the deformation mechanisms must be invoked to establish the correlation.

With regard to cell size, some conflicting conclusions were drawn by different researchers for closed-cell foams. For instance, Yu et al. [26] compared the compressive stress-strain curves of closed-cell aluminium foams and found that plateau stress increases with cell size decreasing. Bouix et al. [34] also reported similar experimental observations for closed-cell polymer foams. They both surmised that the smaller cells are less susceptible to buckling due to their shorter walls when bulk relative density is same, thereby enhancing the load resistance of the cell structures. By contrast, Yu et al. [25] observed that larger cell size leads to higher peak stress, and they explained that larger cells imply thicker and stronger cell walls for a given bulk relative density, which leads to higher load resistance. Similar conflicting conclusions were also drawn for open-cell aluminium foam [33, 47]. The problem of these previous analyses is that they treated mean cell size as an independent structural parameter controlling material properties, but the local structural characterisation was not carried out for the analysed foams. In fact, it is the weakest region in a heterogeneous material that is critical to determine the macroscopic material properties. As it has been widely recognised that the dominant deformation mechanisms for aluminium foams are plastic bending and buckling of cell walls $[1,18,48,49]$, which are also confirmed in this study, the load resistance can be correlated with the structural parameters controlling bending and buckling resistance.

Consider plastic bending, a scaling law can be expressed as [1]

$$
\frac{\sigma_{\mathrm{c}}}{\sigma_{\mathrm{s}}} \propto\left(\frac{h}{s}\right)^{3}
$$

where $\sigma_{\mathrm{c}}$ and $\sigma_{\mathrm{s}}$ are the collapse stress of a unit cell (or assembly of connecting cells) and the yield strength of the cell-wall material, respectively; $h$ and $s$ are cell-wall thickness and cell-wall length, respectively. For buckling, we have [1] 


$$
\frac{\sigma_{\mathrm{c}}}{E_{\mathrm{s}}} \propto\left(\frac{h}{s}\right)^{4}
$$

where $E_{\mathrm{s}}$ is Young's modulus or tangent modulus of cell-wall material.

According to Eqs. (3) and (4), the load bearing capacity of cell walls is mainly determined by the ratio of cell-wall thickness to cell-wall length. The cell-wall length is equal to cell size in the cubic unit cell model proposed by Gibson and Ashby [1], while for the 2D foam studied here, the mean cell-wall length is about $71 \%$ of the mean cell size (i.e. equivalent diameter). Fig. 18b shows that the first crush band forms at the axial location where the $h / s$ is a minimum, indicative of a sufficient condition for the initiation of a crush band. Interestingly, the minimum $h / s$ is also approximately corresponding to the minimum local relative density, see Fig. $18 \mathrm{~b}$ and Fig. 9a. Indeed, Gibson and Ashby [1] found that the relative density is approximately proportional to $(h / s)^{2}$ for many types of foams. However, in a specific foam, the local relative density may have different coefficients in its dependence on local $(h / s)^{2}$. For the 2D foam studied here, the ratio of local relative density to local $(h / s)^{2}$ varies between 1.4 and 2.9 for different through-width sub-regions $(l=3.65 d)$, and this variation is mainly because of the heterogeneity of the cell structure.

The above analyses show that the minimum thickness-to-length ratio of cell walls indicates the "weakest" region in the 2D foam. However, in some other cellular materials, the orientation and anisotropy of cells or the inclination and straightness of cell walls may be much more heterogeneous, and then they could play more important roles in the cell collapse, depending on the specification of the cellular material. On the other hand, when the cell structure is homogeneous at the meso-scale, e.g. periodically arrayed cells in honeycombs and Kelvin foams, a shear band with a considerable inclination angle can be initiated and its formation is sensitive to the boundary condition and sample size [50].

Most importantly, the discussion presented above shows that, for heterogeneous cellular materials, the characterisation of meso-scale cell structure and the measurement of local structural parameters are necessary and crucial for establishing the correlation between the meso-scale structure and deformation, thereby identifying the "weakest" region which 
determines the collapse or failure stress of the cellular material.

\section{Conclusions}

A 2D foam was constructed from a CT slice image of a closed-cell aluminium Alporas ${ }^{\mathrm{TM}}$ foam sample and characterised by meso-scale structural parameters including the size, aspect ratio, orientation and anisotropy of cells, as well as the length, inclination, straightness and thickness of cell walls. A virtual quasi-static compression test was performed for the 2D foam using image-based finite element simulation, and localised cell collapse and crushing were characterised via cell-wall strain, cell area ratio, DIC strain and cross-sectional engineering strain. Local structural parameters were averaged laterally in the sub-regions perpendicular to the compression axis. It was demonstrated that the average (lower bound) and the maximum (upper bound) of cell size can be used as two spatial thresholds for characterising the meso-scale heterogeneity of structure and deformation in a foam material. It was found that the cell structure of the 2D foam has no evidently preferred orientation or significant anisotropy. The morphological parameters such as the aspect ratio, orientation and anisotropy of cells and the straightness and inclination of cell walls are relatively uniformly distributed in the early collapsed region. However, the local relative density, cell-wall thickness, cell-wall length and cell size exhibit considerable meso-scale heterogeneity. As the plastic bending and buckling of the cell walls are dominant deformation modes, it was identified that the sufficient condition for the initiation of a crush band is a minimum of the ratio of cell-wall thickness to cell-wall length averaged in the through-width sub-regions, rather than the individual values of cell-wall length (or equivalently, cell size) and cell-wall thickness. This study shows that the local structural characterisation is necessary to reveal the effects of heterogeneous cell structure on the meso-scale localised deformation and the overall load resistance, which are controlled by the "weakest" region in the cell structure.

Acknowledgements: The first author is grateful for the assistance given by the IT Services and the use of the Computational Shared Facility at The University of Manchester (UoM). The fourth author acknowledges the support from National Science Foundation of China (No.11272060).

\section{References}

[1] L.J. Gibson, M.F. Ashby, Cellular solids: structure and properties, 2 ed., Cambridge University Press, 1997.

[2] L.P. Lefebvre, J. Banhart, D.C. Dunand, Advanced Engineering Materials, 10 (2008) 775-787.

[3] M.F. Ashby, A.G. Evans, N.A. Fleck, L.J. Gibson, J.W. Hutchinson, H.N.G. Wadley, Metal foams: a design 
guide, Elsevier, 2000.

[4] M. Saadatfar, M. Mukherjee, M. Madadi, G.E. Schröder-Turk, F. Garcia-Moreno, F.M. Schaller, S. Hutzler, A.P. Sheppard, J. Banhart, U. Ramamurty, Acta Materialia, 60 (2012) 3604-3615.

[5] A.H. Benouali, L. Froyen, T. Dillard, S. Forest, F. N'guyen, Journal of Materials Science, 40 (2005) 5801-5811.

[6] A.E. Simone, L.J. Gibson, Acta Materialia, 46 (1998) 3109-3123.

[7] E. Andrews, W. Sanders, L.J. Gibson, Materials Science and Engineering A, 270 (1999) 113-124.

[8] J. Němeček, V. Králík, J. Vondřejc, Computers \& Structures, 128 (2013) 136-145.

[9] T. Hildebrand, P. Rüegsegger, Journal of Microscopy, 185 (1997) 67-75.

[10] M.K. Khan, T. Baig, S. Mirza, Materials Science and Engineering: A, 539 (2012) 135-142.

[11] S.D. Papka, S. Kyriakides, Journal of the Mechanics and Physics of Solids, 42 (1994) 1499-1532.

[12] W.-Y. Jang, S. Kyriakides, International Journal of Solids and Structures, 46 (2009) 617-634.

[13] Q.M. Li, R.A.W. Mines, Strain, 38 (2002) 132-140.

[14] A. Wilbert, W.Y. Jang, S. Kyriakides, J.F. Floccari, International Journal of Solids and Structures, 48 (2011) 803-816.

[15] D. Mohr, M. Doyoyo, Journal of Applied Physics, 94 (2003) 2262-2270.

[16] A.T. Barnes, K. Ravi-Chandar, S. Kyriakides, S. Gaitanaros, International Journal of Solids and Structures, 51 (2014) 1631-1645.

[17] L. Wang, Y. Ding, L. Yang, International Journal of Impact Engineering, 62 (2013) 48-59.

[18] H. Bart-Smith, A.F. Bastawros, D.R. Mumm, A.G. Evans, D.J. Sypeck, H.N.G. Wadley, Acta Materialia, 46 (1998) 3583-3592.

[19] M.A. Sutton, W.J. Wolters, W.H. Peters, W.F. Ranson, S.R. McNeill, Image Vision Comput, 1 (1983) $133-139$.

[20] P. Bing, Q. Kemao, X. Huimin, A. Anand, Measurement Science and Technology, 20 (2009) 062001.

[21] F. Pierron, S.A. McDonald, D. Hollis, P.J. Withers, A. Alderson, Applied Mechanics and Materials, 70 (2011) 93-98.

[22] Z. Zou, S.R. Reid, P.J. Tan, S. Li, J.J. Harrigan, International Journal of Impact Engineering, 36 (2009) 165-176.

[23] S. Liao, Z. Zheng, J. Yu, International Journal of Solids and Structures, 51 (2014) 478-490.

[24] C. Chen, T.J. Lu, N.A. Fleck, Journal of the Mechanics and Physics of Solids, 47 (1999) 2235-2272.

[25] H. Yu, Z. Guo, B. Li, G. Yao, H. Luo, Y. Liu, Materials Science and Engineering: A, 454-455 (2007) $542-546$.

[26] J.L. Yu, J.R. Li, S.S. Hu, Mechanics of Materials, 38 (2006) 160-170.

[27] L. Tang, X. Shi, L. Zhang, Z. Liu, Z. Jiang, Y. Liu, Acta Mechanica, 225 (2014) 1361-1372.

[28] Z. Li, J. Zhang, J. Fan, Z. Wang, L. Zhao, Mechanics of Materials, 68 (2014) 85-94.

[29] L. Li, P. Xue, Y. Chen, H.S.U. Butt, Materials Science and Engineering: A, 636 (2015) 60-69.

[30] A.E. Simone, L.J. Gibson, Acta Materialia, 46 (1998) 3929-3935.

[31] A.E. Simone, L.J. Gibson, Acta Materialia, 46 (1998) 2139-2150.

[32] S.A. McDonald, P.M. Mummery, G. Johnson, P.J. Withers, Journal of Microscopy, 223 (2006) 150-158.

[33] B. Jiang, Z. Wang, N. Zhao, Scripta Materialia, 56 (2007) 169-172.

[34] R. Bouix, P. Viot, J.-L. Lataillade, International Journal of Impact Engineering, 36 (2009) 329-342.

[35] J. Banhart, Progress in Materials Science, 46 (2001) 559-632.

[36] Y. Sun, Q.M. Li, S.A. McDonald, P.J. Withers, Mechanics of Materials, 99 (2016) 26-36.

[37] I. Jeon, T. Asahina, Acta Materialia, 53 (2005) 3415-3423.

[38] Y. Sun, Q.M. Li, International Journal of Solids and Structures, 63 (2015) 50-67. 
[39] Y.D. Liu, J.L. Yu, Z.J. Zheng, J.R. Li, International Journal of Solids and Structures, 46 (2009) 3988-3998.

[40] Z. Zheng, J. Yu, J. Li, International Journal of Impact Engineering, 32 (2005) 650-664.

[41] T. Zhang, C.Y. Suen, Communications of the ACM, 27 (1984) 236-239.

[42] I. Arganda-Carreras, R. Fernández-González, A. Muñoz-Barrutia, C. Ortiz-De-Solorzano, Microscopy Research and Technique, 73 (2010) 1019-1029.

[43] P.G. Young, T.B.H. Beresford-West, S.R.L. Coward, B. Notarberardino, B. Walker, A. Abdul-Aziz, Philosophical Transactions of the Royal Society A: Mathematical, Physical and Engineering Sciences, 366 (2008) 3155-3173.

[44] U. Ramamurty, A. Paul, Acta Materialia, 52 (2004) 869-876.

[45] J. Quinta Da Fonseca, P.M. Mummery, P.J. Withers, Journal of Microscopy, 218 (2005) 9-21.

[46] A.G. Hanssen, O.S. Hopperstad, M. Langseth, H. Ilstad, International Journal of Mechanical Sciences, 44 (2002) 359-406.

[47] H. Kanahashi, T. Mukai, T. Nieh, nbsp, G, T. Aizawa, K. Higashi, Mater Trans, 43 (2002) 2548-2553.

[48] Y.L. Sun, T. Lowe, S.A. McDonald, Q.M. Li, P.J. Withers, In Situ Investigation and Image-Based Modelling of Aluminium Foam Compression Using Micro X-Ray Computed Tomography, Visual Computing: Scientific Visualization and Imaging Systems, Springer, 2014, pp. 189-197.

[49] J. Zhou, P. Shrotriya, W.O. Soboyejo, Mechanics of Materials, 36 (2004) 781-797.

[50] W.-Y. Jang, S. Kyriakides, International Journal of Solids and Structures, 46 (2009) 635-650. 